\title{
Wind Turbine Gearbox Failure and Remaining Useful Life Prediction Using Machine Learning Techniques.
}

James Carroll*, Sofia Koukoura, Alasdair McDonald, Anastasis Charalambous Stephan Weiss and Stephen McArthur

All Authors: Electronic and Electrical Engineering, University of Strathclyde, Glasgow, Scotland *Corresponding Author Email: j.carroll@strath.ac.uk

*Corresponding Author Address: Room 336, EEE Department, University of Strathclyde, Glasgow, G1 1XD

\section{ABSTRACT}

This research investigates the prediction of failure and remaining useful life (RUL) of gearboxes for modern multi-megawatt wind turbines. Failure and RUL are predicted through the use of machine learning techniques and large amounts of labelled wind turbine SCADA and vibration data. The novelty of this work stems from unprecedented access to one of the world's largest wind turbine operational and reliability databases, containing thousands of turbine gearbox failure examples and complete SCADA and vibration data in the build up to those failures. Through access to that data this paper is unique in having enough failure examples and data to draw the conclusions detailed in the reminder of this abstract.

This paper shows that artificial neural networks provide the most accurate failure and RUL prediction out of three machine learning techniques trialled. This work also demonstrates that SCADA data can be used to predict failure up to a month before it occurs and high frequency vibration data can be used to extend that accurate prediction capability to 5-6 months before failure. This paper demonstrates that two class neural networks can correctly predict gearbox failures between 72.5 and $75 \%$ of the time depending on the failure mode when trained with SCADA data and $100 \%$ of the time when trained with vibration data. Data trends in the build up to failure and weighting of the SCADA data inputs are also provided. Lastly, this work shows how multi-class neural networks demonstrate more potential in predicting gearbox failure when trained with vibration data as opposed to training with SCADA data.

Keywords: Failure, Remaining Useful Life, Prediction, Wind Turbine, Gearbox, Machine Learning, Vibration, SCADA 


\section{Introduction}

The cost of energy $(\mathrm{CoE})$ for offshore wind is currently too high to make it truly competitive with traditional fossil fuel energy generation techniques. ${ }^{1}$ Past analysis has shown that costs for offshore wind energy are roughly $40 \%$ higher than gas turbine generation and $30 \%$ higher than onshore wind. ${ }^{2}$ Consequently, industry, research institutes and academia are working towards lowering the $\mathrm{CoE}$ for offshore wind. High operation and maintenance $(\mathrm{O} \& \mathrm{M})$ costs present an opportunity to lower this CoE. O\&M costs can be up to $30 \%$ of the overall $\mathrm{CoE}$ for some offshore wind farms ${ }^{3}$ and it is expected that this could further increase in the coming years as wind farms move further offshore and into harsher environments.

Both downtime and costs - as a consequence of poor maintenance planning - can be reduced through an ability to predict if and when wind turbine components will fail. Data analytics, feature extraction and machine learning techniques show promising potential to achieve this. ${ }^{4-10}$ The gearbox has one of the highest downtimes and replacement costs out of all wind turbine components. ${ }^{11}$ Consequently, trying to predict gearbox failure has been the focus of a number of past papers. ${ }^{4,6,10,12-21}$ Amongst techniques to predict gearbox failure, there are both model-based ${ }^{13,17}$ and data-driven 4, 7, 8, 19, 22 approaches. In the past, modelbased approaches have utilised a non-linear model of the system ${ }^{13}$ or a temperature model of the gearbox. ${ }^{17}$ The quality of the failure prediction will depend on the complexity and accuracy of the model, which may be adjusted by data-driven components. ${ }^{15}$ Data-driven approaches are typically based on supervisory control and data acquisition (SCADA) measurements and related inputs. ${ }^{6,10,12,15,20}$

For data-driven approaches, reference ${ }^{10}$ outlines a method for detecting wind turbine gearbox and generator failures using SCADA data. It explains how anomaly-detection techniques and multi-agent systems could be used to create a decision support tool for wind turbine operators. The anomaly detection techniques used in ${ }^{10}$ model predicted gearbox oil and bearing temperature based on other SCADA data inputs, specifically, power generated and nacelle temperature. Expected gearbox and oil temperatures were modeled for given power generated and nacelle temperature readings. If the actual values varied by a certain amount from the modeled value an anomaly was detected. 
Data-driven methods are typically trained to extract features from data and execute a classification by machine learning techniques such as artificial neural networks (ANNs). ${ }^{12,15,20}$ Since often a significant number of parameters have to be adjusted, the size of the data set available for training is important. The lack of large data sets is further exacerbated by the need to isolate a test set from the data, which is used to assess the performance of the method and must be separate from the training set. One way that has been attempted to combat this has been to utilize fast-adaptation for ANNs in order to be able to reduce the size of the training data set. $^{15}$

In this paper, we address the detection of gearbox failure and remaining useful life predictions through the use of large amounts of operational and reliability data. This data comprises of SCADA, but additionally includes high frequency vibration data, O\&M orders and logs. This data is used to train and test machine learning algorithms to predict failure. High frequency vibration data has proved useful in the prediction of gearbox failures in automotive applications. ${ }^{23,24}$

The analysis presented in this paper rests on three novel aspects: (i) the large data set on which the analysis is based; (ii) the inclusion of high-frequency vibration data into the failure and RUL prediction of gearboxes specifically for wind turbines; and (iii) the availability of a significantly higher number of failure cases than had previously been reported elsewhere. On the latter point, past papers ${ }^{5,10,20,25}$, all relied on less than 5 failure examples for their training, while this work relies on hundreds of failure cases from thousands of turbines. Based on this data and methodology for predicting the failure and remaining useful life of wind turbine gearboxes, enough examples of the exact same failure mode exist to allow for failure mode specific remaining useful life predictions, which have not been reported in the literature previously.

\section{Method}

\subsection{Overview}

The methods used to predict failure and remaining useful life typically require large amounts of failure examples and wind turbine operational data to train and test the machine learning algorithms. A leading wind turbine manufacturer provided full access to their operational logs, failure logs, SCADA data and vibration 
data from the tens of thousands of turbines they monitor located in thousands of wind farms globally. Due to the gearbox being the sub-system of the turbine that has the highest failure cost associated with it ${ }^{5}$, the industrial partners agreed that it should be the component that is the focus of this work. Working with the wind turbine manufacturer, a large number of the same type of gearbox failures from the same type of gearbox model were identified. Details of these failure modes can be seen in Section 2.2. Using the failure logs the date of each failure was recorded and SCADA data and vibration data from various time periods in the build up to failure were identified, obtained and labeled. Once the SCADA data and vibration data was obtained it was preprocessed and plotted to qualitatively determine how it changed at various points in the build up to failure. The SCADA and vibration data was then used to test and train the machine learning algorithms. Different machine learning algorithms and training groups were then tested to determine the algorithm and training method that provided the most accurate failure and remaining useful life predictions. Weightings of the inputs were determined and conclusions were then drawn on the possibility of predicting failure and remaining useful life using machine learning techniques tested and trained with SCADA and Vibration data. The following 6 steps provide a high level overview of each of the work packages required to complete this research. The following sub-sections then provide further details on each of the steps outlined below. Step 2 aligns with Subsection 2.2, step 3 aligns with Subsection 2.3 and so on up to step 5.

Step 1: Obtain Access to the operational data of a major wind turbine manufacturer. Step 2: Identify a population of failures from the same gearbox model and same failure mode.

Step 3: Obtain the SCADA data and vibration data in the build up to failures from the populations identified in Step 2.

Step 4: Pre-process and plot the SCADA and vibration data in the build up to failures.

Step 5: Train and test different machine learning algorithms using different training groups and determine weightings.

Step 6: Draw conclusions on the use of machine learning algorithms trained with SCADA and vibration data for predicting failure and RUL. 


\subsection{Failure modes and Population details}

This sub-section provides further details on Step 2 from Section 2.1. When selecting populations of failure modes and gearbox models to use in this work, two gearbox models that encountered repeated failures were chosen. Both of the gearbox models include a low speed planetary stage, an intermediate stage and a high speed parallel stage (Figure 1). The two gearbox models come from a different turbine type. Both gearbox models are used in multi-MW scale turbines with a rating of between $2 \mathrm{MW}$ and $4 \mathrm{MW}$ and a rotor diameter of between 80 and 120 metres. Both turbine types use high speed gearboxes with induction generators. The failure examples in this paper come from over 50 different wind farms located throughout Europe. Further details on the turbine types cannot be provided for confidentiality reasons.

All gearboxes that encounter the gear bearing issue are the same gearbox model and for the same turbine type ('A'). Similarly, all gearboxes that encounter the gear tooth issue are the same gearbox model and for the same turbine type ('B').

Both gearbox configurations and locations of where the vibration readings were obtained are seen in Figure 1. Gearbox "A" has two planetary stages and one parallel stage and gearbox type "B" has one planetary stage and two parallel stages:
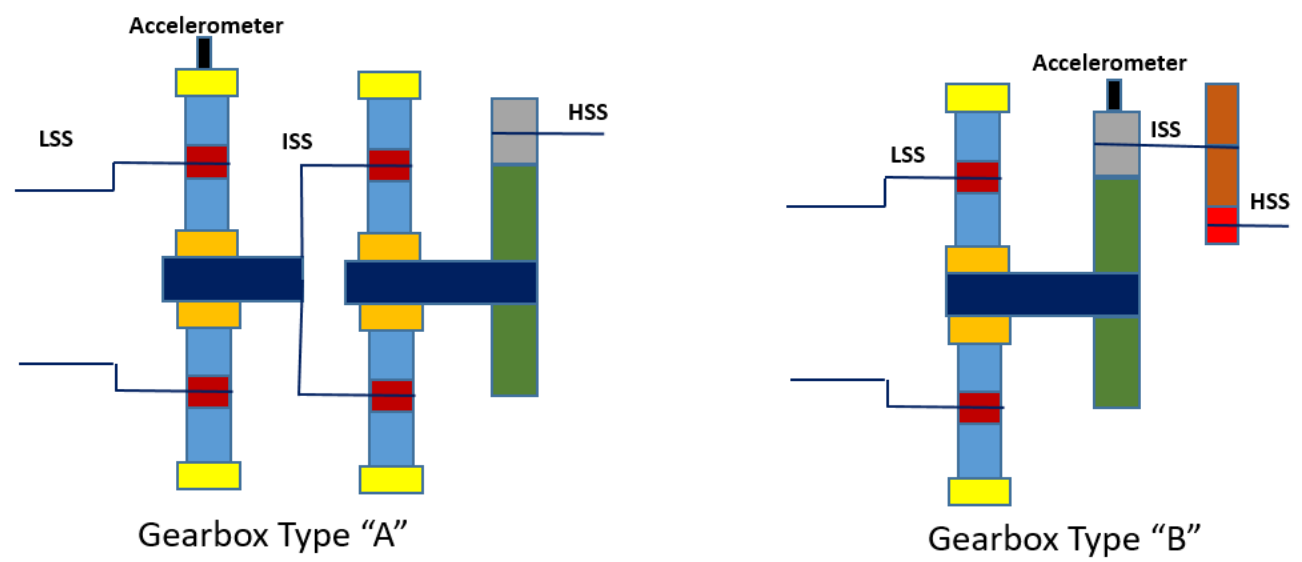

Figure 1. Gearbox configurations and location of accelerometers 
For the purpose of this analysis the date of failure is defined as the date at which the wind turbine was stopped for the gearbox to be replaced or repaired. As far as the author is aware these repairs and replacements were carried out due to critical failures rather than due to the predicted possibility of a critical failure. In this case, components reached the point of critical failure because the condition monitoring system used did not pick up the possibility of failure in advance.

\subsubsection{Gearbox Bearing Issue}

The gearbox planet bearing issue is located on the low speed planetary stage of the gearbox. The bearing issue eventually results in complete failure of the bearing and subsequently the gearbox. When this occurs the turbine is shut down and only restarted once a complete gearbox exchange occurs. For training and testing of the machine learning algorithms in this work, the data from 200 different examples of this failure were obtained from turbine type A and gearbox model A. Figure 2 shows a borescope image of the bearing in the lead up to failure in which indents in the raceway and rolling element can be seen.

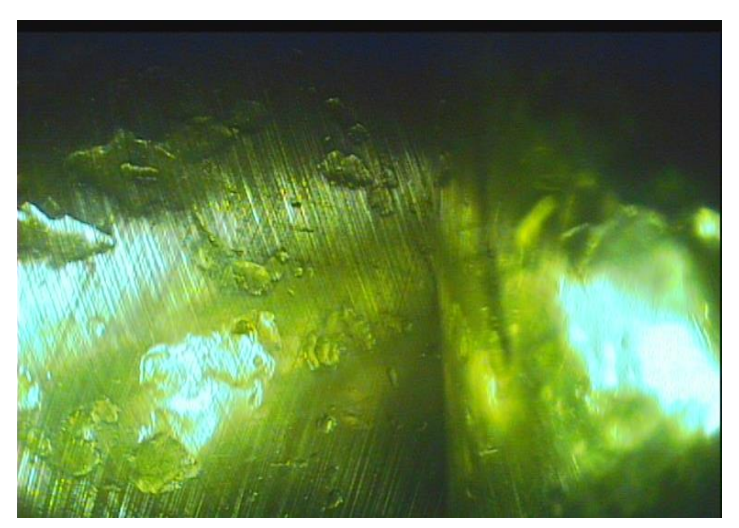

Figure 2. Borescope image of bearing issue showing indents

\subsubsection{Gear Tooth Issue}

The gearbox tooth issue is located on the pinion of the intermediate stage of the gearbox. The tooth issue eventually results in complete failure of the intermediate stage of the gearbox. When this occurs the turbine is shut down and only restarted once an exchange of the intermediate gearbox stage occurs. For training and testing of the machine learning algorithms in this work, the data from 28 different examples of this failure 
were obtained from turbine type B and gearbox model B. Figure 3 shows a borescope image of the gear tooth in the lead up to failure in which the damaged pinion tooth can be seen.

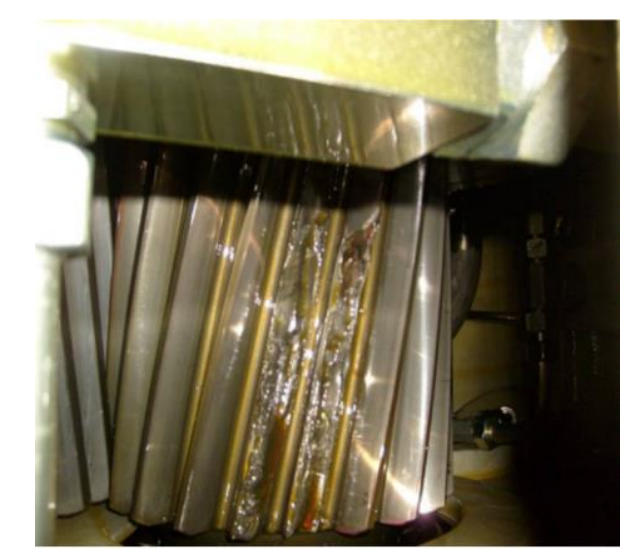

Figure 3. Borescope image of pinion tooth damage

\subsection{Obtain SCADA and Vibration Data}

In the wind turbine manufacturer's operational data centre a number of different databases exist. Once a failure date was identified for a certain gearbox in one database, a second database was accessed to get the SCADA data in the build up to that failure. This process was carried out for each failure in both the gearbox bearing issue population and the gear tooth issue population. The SCADA data was exported from the wind turbine manufacturer's data center using SQL. As the vibration data is such high frequency data, it is stored in a different manner to the SCADA data. It is located on a server in a text file format for ease of storage. The required vibration files were found through the wind farm name and turbine ID and subsequently downloaded.

\subsection{SCADA and Vibration Data Pre-processing and Trending}

This sub-section provides further details on Step 4 from Section 2.1, the pre-processing of the SCADA data and the vibration data. In this research, monthly intervals were used when describing the time to failure for both the SCADA and vibration data. The authors and industrial partner decided to work with monthly intervals following an early discussion about the industrial partner's needs in terms of failure warning periods for maintenance planning. Following that discussion, it was agreed that for maintenance planning reasons (in this particular case) knowledge that a component would fail in the next month was equally as useful as knowing it would fail in the next week or day. For that reason, monthly periods were chosen. 


\subsubsection{SCADA data pre-processing and Trending}

SCADA data from a number of periods before failure were taken. One thousand and eight, ten-minute averaged data points per sensor, or one week's worth of data, from 1 month, 2 months, 3 months, 6 months, 1 year and 1.5 years before failure were obtained. Once the data was obtained, the SCADA data quality was checked to ensure no large periods were missing. After the SCADA data quality was confirmed, the sensor temperature readings were subtracted from each other to provide differences in temperature $(\Delta T)$ to train the machine learning algorithms. For example, directly from the SCADA system a gear oil temperature reading ( $\left.T_{\mathrm{oil}}\right)$ and an ambient temperature reading $\left(T_{\mathrm{amb}}\right)$ were obtained, and the difference in temperatures was found $\Delta T=T_{\mathrm{oil}}-T_{\mathrm{amb}}$. This $\Delta T$ was considered because if the ambient or nacelle temperature rises significantly then the gearbox oil temperature may also rise even when the gearbox is in a healthy state. To determine the importance of feature engineering (e.g. using the deltas) in this case, a results comparison was carried out when deltas were included and excluded. When the neural network hyper-parameters were not optimized, the number of correct predictions were $3 \%$ higher when deltas were included. However, when hyperparameters were optimized the correct predictions were the same, regardless of whether the deltas were included. As having the deltas included did not decrease the accuracy of the predictions it was decided to include them in this analysis.

The following SCADA data columns were obtained from the bearing issue in gearbox model A/turbine type A. The column headings shown in italic did not come directly from the SCADA system, those data points are the deltas between the readings that came directly from the SCADA system (shown as non-italic headings). All readings including the temperature deltas resulted in 27 inputs for the training of the machine learning algorithms for the gearbox bearing issue.

1. Gear Oil Temperature (Sensor 1)

2. Gear Oil Temperature (Sensor 2)

3. $\Delta T$ Oil Sensor 1 and Oil Sensor 2 4. 4 T Oil Sensor 1 and Nacelle 5. $\Delta T$ Oil Sensor 1 and Ambient
6. $\Delta T$ Oil Sensor 2 and Nacelle

7. $\Delta T$ Oil Sensor 2 and Ambient

8. Gear Bearing 1 Temperature

9. Gear Bearing 2 Temperature

10. Gear Bearing 3 Temperature 
11. Gear Bearing 4 Temperature

12. Gear Bearing 5 Temperature

13. $\Delta T$ Bearing 1 and Nacelle

14. $\Delta T$ Bearing 1 and Ambient

15. $\Delta T$ Bearing 2 and Nacelle

16. $\Delta T$ Bearing 2 and Ambient

17. $\Delta T$ Bearing 3 and Nacelle

18. $\Delta T$ Bearing 3 and Ambient

19. $\Delta T$ Bearing 4 and Nacelle
20. $\triangle T$ Bearing 4 and Ambient

21. $\Delta T$ Bearing 5 and Nacelle

22. $\Delta T$ Bearing 5 and Ambient

23. Nacelle Temperature

24. Rotor Speed

25. Wind Speed

26. Ambient Temperature

27. Total Power Production

The same procedure was followed for the gear tooth issue. However, Turbine Type B's SCADA system is less advanced than type A. It consists of less sensors around the gearbox resulting in less direct readings and less temperature deltas. In total 12 input columns were obtained for testing and training the machine learning algorithms.

1. Generator Speed

2. Gear Oil Temperature Sensor

3. $\Delta T$ Oil Sensor and Nacelle

4. $\Delta T$ Oil Sensor and Ambient

5. Gear Bearing Temperature

6. $\Delta T$ Gear Bearing and Nacelle
7. $\Delta T$ Gear Bearing and Ambient

8. Nacelle Temperature

9. Rotor Speed

10. Wind Speed

11. Ambient Temperature

12. Total Power Production

The SCADA temperature data was then plotted to determine the trends in the data in the build up to failure.

These plots can be seen in Section 3.1 .

\subsubsection{Vibration data pre-processing and trending:}

As mentioned in the previous step the vibration data was obtained from the industrial partner in a text file format. Vibration data from a number of periods before failure were taken. One text file was taken each week in the build up to failure. As the accelerometers were providing data above $25 \mathrm{kHz}$ and each file contained 10 seconds of data, over 250,000 readings were provided per accelerometer. Each file contains data from 10 different accelerometers or tachometers from various locations on the drivetrain, so each 10 second file has over 2,500,000 data points. In the case of the intermediate stage pinion tooth issue, the intermediate stage accelerometer and tachometer were the focus of the work. The 256,000 vibration data readings were imported to MATLAB and through the application of Fast Fourier Transform (FFT) and Order 
Tracking functions the spectrum plots shown in Figure 4 and Figure 10 were created (order tracking accounts for the varying rotational speed in modern variable speed wind turbines). The vibration data from various time instances before the failure were consider and plotted to show the vibration trends in the build up to failure. A picture emerges of the side bands rising around the gear meshing frequency as the time axis gets closer to the point for failure. The resulting plot can be seen in Section 3.2, Figure 10. A similar process for the bearing issue was followed, however the trends toward failure were not clear when an FFT was applied. For that reason and based on ${ }^{26}$ an envelope analysis was carried out. Even with the envelope analysis, clear trends were not seen around the ball passing frequencies on the bearing issue.

To obtain inputs for training the machine learning algorithms with the vibration data, magnitude readings were taken from the spectrum. Figure 4 shows the spectrum from one time period in the build up to failure of the gear tooth. The rising sidebands can be seen around the gear meshing frequency. The red lines on Figure 4 indicates the points on the spectrum where magnitude readings were taken (indicated in red, above the spectrum). Past literature suggests that the magnitude and shape of the gear meshing frequency and its side bands is indicative of a gear tooth issue ${ }^{27}$. This was repeated for the $2^{\text {nd }}, 3^{\text {rd }}$ and $4^{\text {th }}$ harmonics, as suggested by ${ }^{27}$.

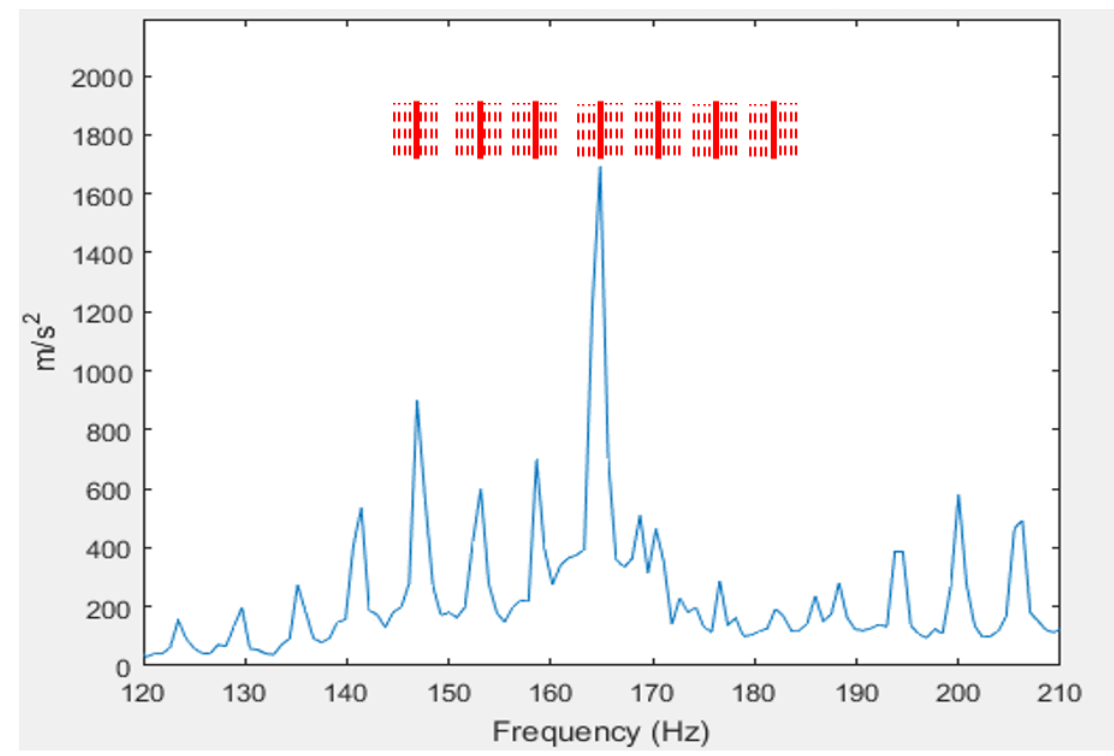

Figure 4. Data points taken from spectrum for training of machine learning techniques. (Figure created using vibration data from gear tooth issue) 
While clear trends are seen around the gear meshing frequency for the gear tooth issue shown in Figure 4, no clear trends around ball passing frequencies were easily identified in the bearing issue. Accordingly, a large number of magnitude readings were taken from the spectral area around the ball passing frequencies. The magnitude readings as shown in Figure 4 and from the bearing issue spectrum were then used to train the machine learning algorithms.

\subsection{Machine learning algorithms selection testing and training}

Three popular machine learning algorithms were trained and tested in this research: an artificial neural network, a support vector machine (SVM) and a logistic regression approach. An analysis (results shown in Section 3.3) compared the number of correct predictions from the three different techniques. The algorithm that provided the highest number of correct predictions then became the focus of the other failure and remaining useful life prediction analyses in this paper.

The labeled SCADA and vibration data detailed in Section 2.4.1 and 2.4.2 were imported to Azure Machine Learning Studio ${ }^{28}$ for algorithm testing and training. In this research the SCADA and vibration data were split, with $70 \%$ used for training and $30 \%$ used for testing of all machine learning algorithms, as in ${ }^{29}$. This SCADA and vibration data split can be seen for the gear bearing issue in Figure 5. The same 70/30 split was used for the gear tooth issue.

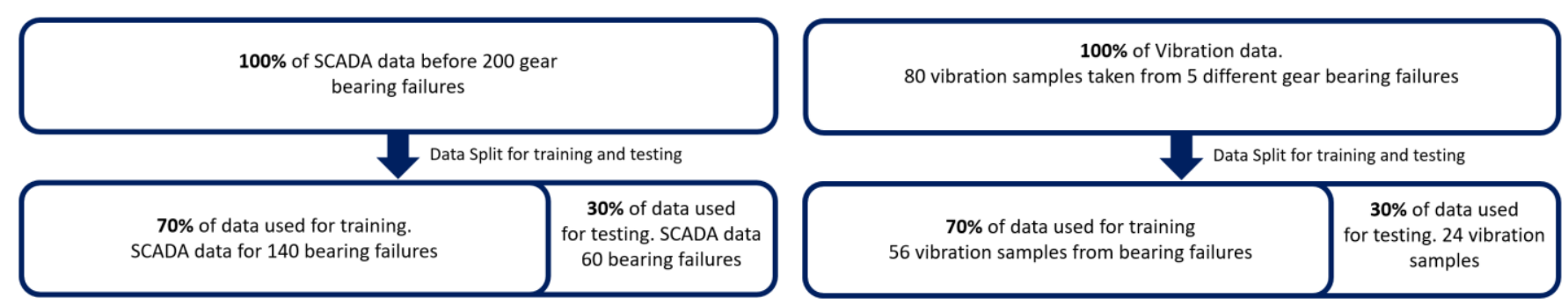

Figure 5. SCADA and Vibration Data Split for Gear Bearing Issue 
The input dimensionality for the SCADA data gear bearing model is the 27 SCADA data readings detailed in Section 2.4.1. The input dimensionality for the SCADA data gear tooth model is the additional 12 SCADA data readings detailed in Section 2.4.1.

The input dimensionality for the vibration data gear tooth model is the 63 magnitude readings from the spectrum (after an FFT has been carried out on the raw vibration data as shown in Figure 4) at the gear meshing frequencies and the sidebands of the gear meshing frequencies. 63 magnitude readings are taken from the spectrum for the first, second, third and fourth gear meshing frequency giving a total of 252 inputs. The input dimensionality for the vibration data gear bearing model is the 30 magnitude readings either side of the ball passing frequency spread at $0.25 \mathrm{~Hz}$ spacing. With 30 readings either side of the ball passing frequency a total of 60 magnitude readings are taken for each ball passing frequency. In total, magnitude readings are taken from the spectrum for the first 4 ball passing frequencies (as for the gear tooth issue) giving a total of 240 inputs.

In both vibration examples mentioned above, magnitude readings are taken from the spectrum around frequencies of interest and used as training inputs to allow the algorithms to learn the behavior of important parts of the spectrum in labeled healthy and unhealthy data.

A number of different algorithm hyper-parameters were used in the training of the neural networks, SVMs and logistic regression algorithms. To optimize the results of this work the hyper-parameters were tuned.

In the machine learning software used for this analysis (Azure Machine Learning Studio) the function used to tune the hyper-parameters is called "Tune Model Hyper-parameter". To tune the hyper-parameters this function tests multiple models, using different combinations from a grid of inputs, and compares metrics over all models to get the optimum combination of settings. For this case, the optimum combination of settings is determined by the highest number of correct predictions. To tune the model, this function performs a parameter sweep over the entire grid of parameter settings, and learns the optimum inputs to obtain the highest number of correct predictions. 
With the optimized hyper-parameters, shown below, and the raw data used in this analysis, the results in this paper can be replicated in Azure Machine Learning Studio.

The following optimized hyper-parameters were used in the training of the two-class and multi-class artificial neural networks. Further detail from each parameter can also be found in ${ }^{28}$

- Number of hidden nodes: The mean of the number of inputs and number of outputs.

- Learning rate: 0.08

- Number of learning iterations: 400

- Initial learning weight: 0.1

- Momentum: 0

Normalizer: Min-max normalizer

The training procedure for the neural network employs stochastic gradient descent using back propagation as a gradient computing technique. The learning rate input determines the size of the step that is used in stochastic gradient descent. The loss function used in this case is the cross-entropy loss function.

The following parameters were used in the training of the two-class SVM:

- Number of iterations: 100

- Lamda: 0.00001

The following parameters were used in the training of the two-class logistic regression algorithm:

- Optimisation tolerance: $1 \times 10^{-7}$

- L1 regularisation weight: 0.1

- L2 regularisation weight: 0.01

- Memory size for L-BFGS: 50

Following the training of the two-class and multi-class machine learning algorithms with $70 \%$ of the data, the remaining $30 \%$ of the data was used to test the algorithms to determine the number of correct predictions, missed failures and false positives. For the purpose of this paper, a "false positive" is defined as an output labeled as closer to the point of failure than it actually is. For example, an output saying the gear bearing is 1-2 months to failure when in reality it is 5-6 months would be considered a false positive. A number of different data groupings were tested for training an artificial neural network. These groupings are outlined in Section 3.5. 
Weightings of the 27 SCADA data inputs for the bearing issue were determined using a "permutation feature importance" (PFI) function. ${ }^{30}$ The function returns an importance score for each input used in the prediction. The evaluation metric was chosen to be the accuracy of the learning algorithm. The PFI function computes the sensitivity of a model in terms of the evaluation metric to random changes of the input values. Once weightings were established using the PFI function, a backward elimination process was implemented to evaluate the accuracy. More specifically, starting with all the inputs of the model, the feature with the least importance score was pruned in each iteration until all but one of the inputs were removed. This method is discussed in ${ }^{31}$. The accuracy and the new importance scores were estimated in each iteration. The feature importance scores, from higher to lower importance and other results of this weighting analysis are shown in Section 3.6.

\section{Results and Discussion}

\subsection{SCADA data trends}

From a wind turbine operator's perspective, it would be ideal if gearbox bearing and oil temperature trends could be monitored to provide an indication of when a gearbox bearing failure or tooth issue will occur. However, the results shown in Figures 6-9 - which corresponds with the experience provided by the industrial partner - indicate that a simple temperature trending approach alone is rarely successful in highlighting potential failures. Figure 6 is a box plot of one week of SCADA bearing temperature readings from 200 different turbines in the build up to gearbox bearing failures. In total each of the 6 box plot contains $\sim 201,600$ ten-minute data points. With the hypothesis that both failure mechanisms lead to a rise in heat production inside the gearbox, failure should be indicated by a temperature rise. For issue detection to occur through a basic temperature trending technique an operator would need to see a rise in the bearing temperature readings in the months leading up to the failure and a lower temperature in the readings classified as "healthy" (greater than 1.5 years before failure in this case). However as seen in Figure 6, although there is a difference between 
the temperature distributions for the healthy gearboxes and the data for 1-year-to-failure gearboxes - which could potentially indicate fault inception - there appears to be no consistent changes thereafter.

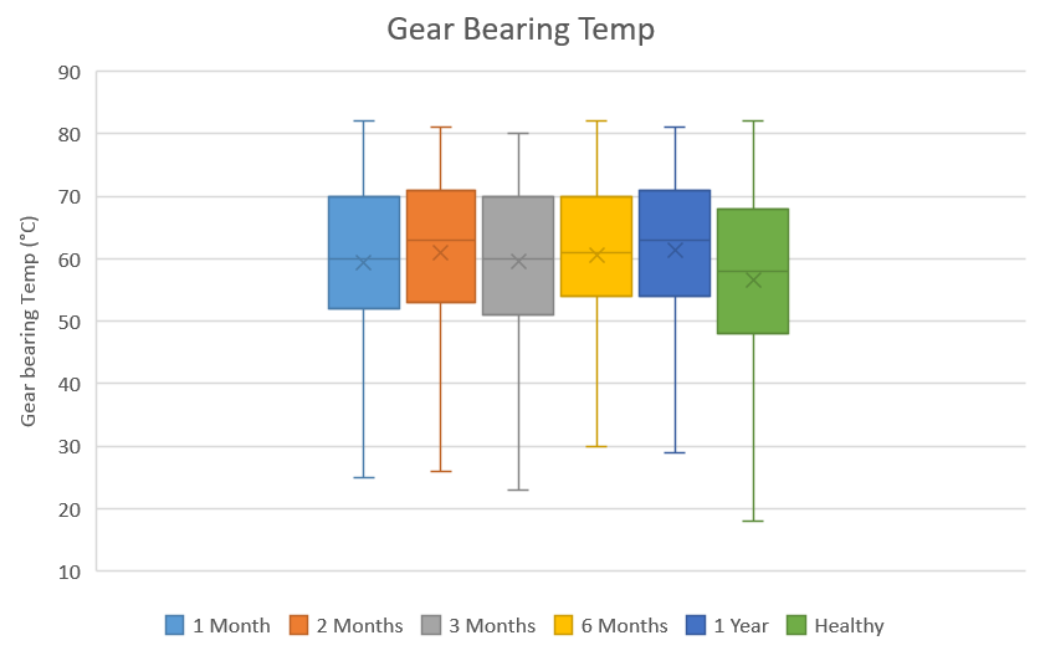

Figure 6. Gear Bearing Temperature at various times before failure. (Figure created using SCADA data from gear bearing issue)

Past papers have suggested a rise in oil temperature in the build up to failure ${ }^{32}$, consequently the authors also looked at the same number of SCADA data reading for the oil temperature in the build up to failure as outlined in the previous paragraph. Once again, there are some differences between healthy and 1-year-tofailure cases, but thereafter no clear trends were observed.

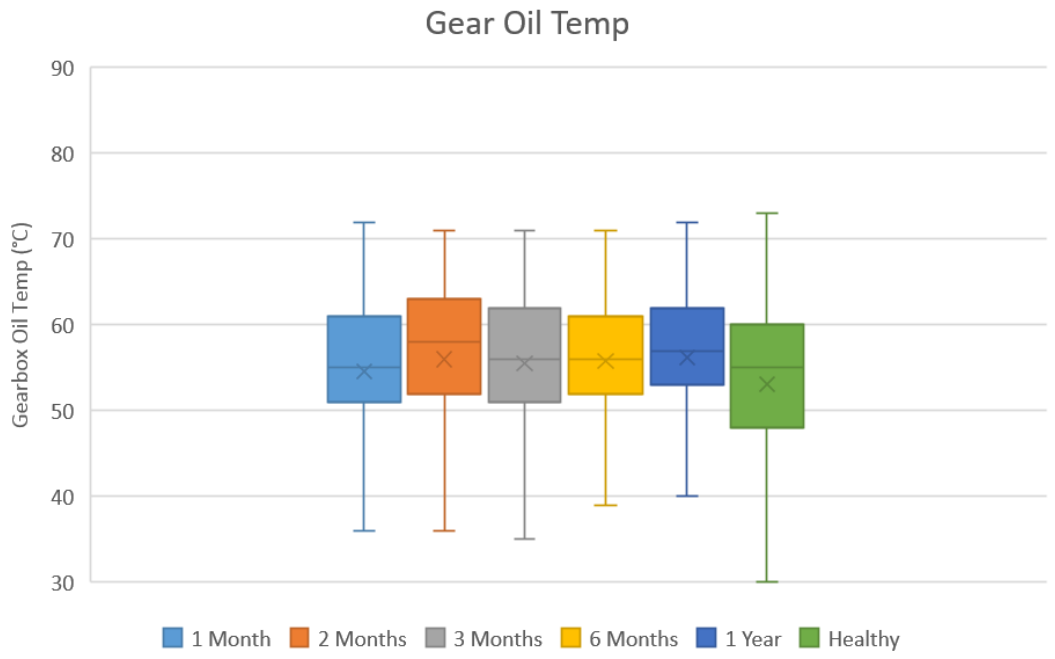

Figure 7. Gear Oil Temperature at various times before failure. (Figure created using SCADA data from gear bearing issue) 
As the ambient temperature could potentially influence the temperature readings in Figure 6 and Figure 7 , the delta between the ambient temperature and gear oil was analyzed and the results are seen in Figure 8 . For example, if the ambient temperature was very low in the month before failure it might mitigate a temperature rise trend presenting itself in Figure 6 and 7. However as seen in Figure 8 the box plots follow the same trend as Figure 7 indicating that gearbox temperature is not greatly influenced by the ambient temperature. The ambient temperature displays little variance in the different time periods to failure.

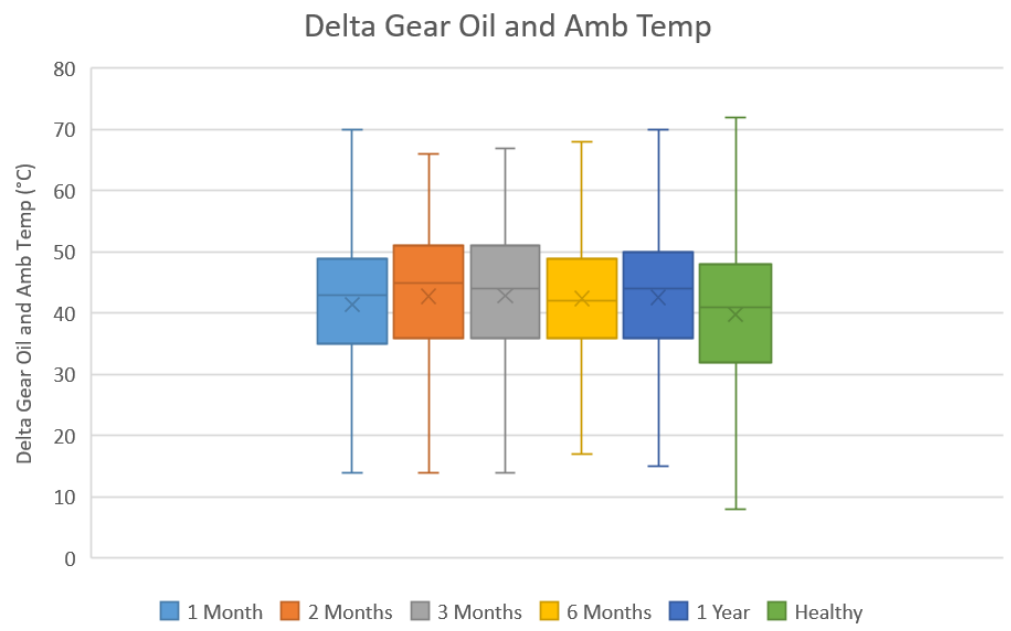

Figure 8. Difference in gear oil and ambient temperatures at various times before failure. (Figure created using SCADA data from gear bearing issue)

The temperature of the gear bearing and gear oil is also influenced by power production. Figure 9 shows the power production for each of the time periods in the build up to failure. When Figure 9 is compared with Figures 6 and 7 a similar trend in the power production and temperatures can be seen which indicates the amount of power produced influences the temperature. For example, "2 months" and "1 Year" have the highest power production and highest temperature readings. While it is expected that the time period before the failure still has an effect on the temperature of the gearbox bearing and gear oil temperature, Figures 6-9 suggest that the influence of power production seems to outweigh the effect of time period to failure. 


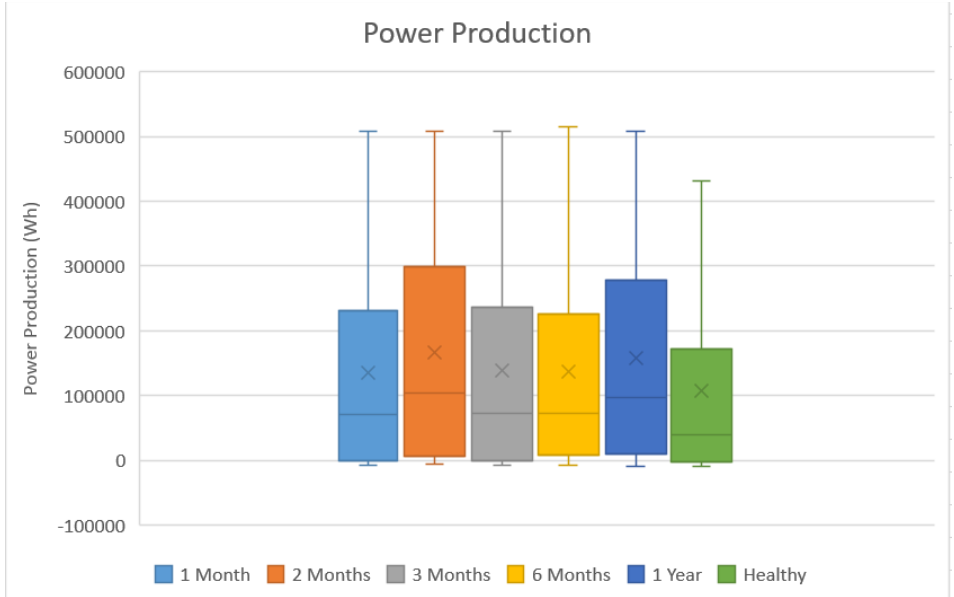

Figure 9. Power Production at various times before failure. (Figure created using SCADA data from gear bearing issue)

Through the analysis of the Figures 6-9, it is clear that other variables outside of time period to failure also influence the temperature of gear bearings and gear oil temperature. For example, gear oil cooling systems will likely reduce the increase in gear oil temperature. A similar analysis to this gear bearing analysis was carried out for the gear tooth issue outlined earlier in this paper and no clear temperature trends were seen in the build up to failure for that failure mode either. As no clear trends are identified, simple temperature trending in isolation may not be enough to correctly identify potential failures due to the influence of other variables. For that reason, a machine learning approach that considers many variables will be used, as demonstrated in Section 3.4.

\subsection{Vibration data trends}

Figure 10 shows the spectrum with order tracking applied to the vibration data at a number of time periods before a gear tooth failure. This technique uses the raw vibration data from the wind turbine's accelerometers and combines it with tachometer data to offset the variable speed of the turbine allowing for a like for like trending analysis in the build up to failure. Order number 6 in Figure 10 is the gear meshing frequency. It is obvious from Figure 10 that a number of sidebands start to rise around the gear meshing frequency as the turbine gets closer to the point of failure. The trend gets clearer once the time period to failure is less than 6 
months. The magnitude of the meshing peaks and side bands are obtained and labeled with their time period to failure to be used in the training of a neural network.

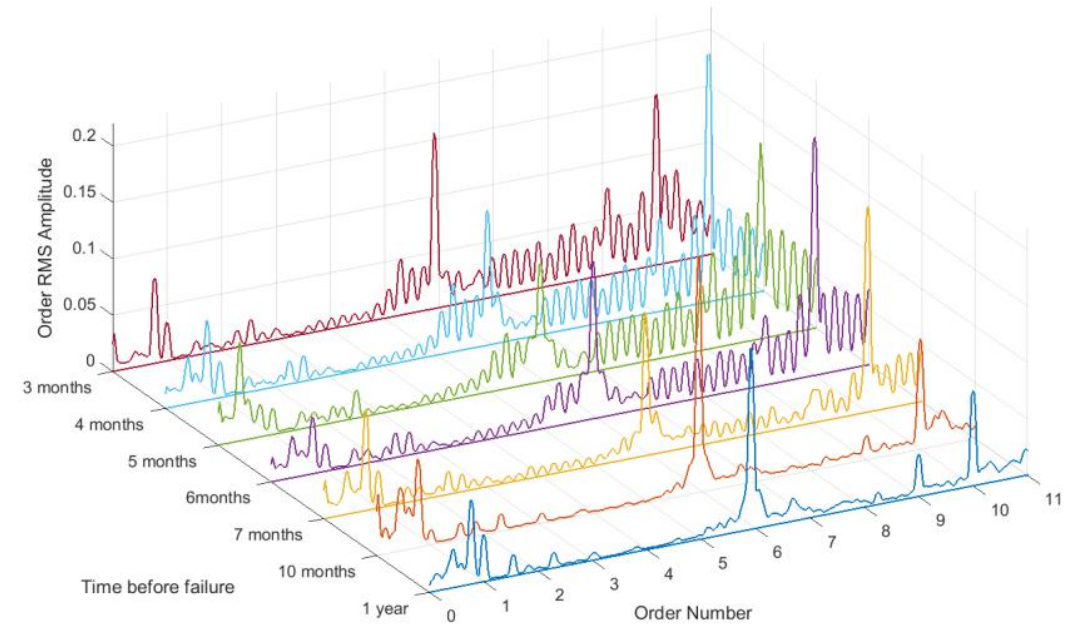

Figure 10. Vibration data showing sidebands developing around the gear meshing frequency (order 6). (Figure created using vibration data from gear tooth issue)

Figure 10 displays the trends for the gear tooth issue in vibration data. A similar analysis was carried out with the vibration data for the bearing issue detailed earlier in this paper. Unlike the gear tooth issue, no visible trends were seen in the gear bearing issue vibration data. The bearing is located in the planetary stage of the gearbox and there is a lot of noise around the ball passing frequencies, leaving it difficult for the authors to pick out failure indicative trends. Consequently $~ 260$ magnitude readings from around the ball passing frequencies of the bearing were obtained for each text file for each bearing failure, labeled with the time period to failure and used to train and test the classification algorithms. Even though no visible trends were clear when the bearing spectrum was plotted, machine learning algorithms could be able to recognize trends in the magnitude readings from the classified vibration data to train a model to be able to classify unlabeled data. This is tested in Section 3.4.

\subsection{Machine learning algorithm selection for gear bearing failures using SCADA input}

To determine which machine learning algorithm provided the most correct health predictions three different two-class classification algorithms were trained and tested with SCADA data for the gear bearing issue. The 
three algorithms considered are the artificial neural network, a SVM and logistic regression. The 27 SCADA data inputs used are outlined in Section 2.4.1. Following the training and testing procedure described in Section 2.5, the results shown in Table 1 were obtained. A correct prediction means that the algorithm correctly stated whether the gearbox bearing would fail in less than one month or more than one month. A missed failure means the algorithm predicted the gearbox would not fail in the next month but the data came from a gearbox that did fail in that month. A false positive means the algorithm predicted the failure would occur in the next month when in reality it did not.

It can be seen that out of the three algorithms tested, the artificial neural network provides the most correct failures and least missed failures, with $72.5 \%$ of failures being correctly predicted. The support vector machine trained and tuned in this analysis used a linear kernel and produced $60 \%$ correct predictions. This correct prediction rate may be increased through the use of a different kernel. Whilst the logistic regression had a similar number of correct predictions to the SVM, the SVM showed fewer false positives but more missed failures than Logistic Regression.

\begin{tabular}{|c|c|c|c|}
\hline & $\begin{array}{c}\text { Artificial Neural } \\
\text { Network }\end{array}$ & $\begin{array}{c}\text { Support Vector } \\
\text { Machine }\end{array}$ & Logistic Regression \\
\hline Correct Prediction & $72.5 \%$ & $60 \%$ & $59 \%$ \\
\hline Missed Failure & $20 \%$ & $36 \%$ & $31 \%$ \\
\hline False Positive & $7.5 \%$ & $4 \%$ & $10 \%$ \\
\hline
\end{tabular}

Table 1: Results of machine learning algorithm comparison for gear bearing issue

As the artificial neural network provided the highest number of correct predictions it became the focus of the remainder of the paper.

\subsection{Two-class neural network predictions for gear bearing and gear tooth failures, using both SCADA and vibration data}

Table 2 shows the results from the training of a two-class neural network for both the gear bearing and gear tooth issue using both SCADA data and vibration data to predict if those failure modes will occur within the next month or outside of the next month, as outlined in Section 2.5. It can be seen in Table 2 that the artificial 
neural network is very successful in predicting a gear tooth failure in the next month using vibration data, and successful $72.5 \%$ of the time at predicting a gear bearing issue in the next month using SCADA data.

The number of correct predictions start to increase slightly for the gear tooth issue when using SCADA data. The gear bearing failure predictions based on vibration data show the lowest number of correct predictions. As discussed in sub-section 3.2, the bearing issue is located in the planetary stage of the gearbox where there is a lot of noise around the ball passing frequencies. Feature extraction on the planetary stage vibration data is an area of further work for the authors and it is hoped improvement will lead to a higher number of correct predictions from the artificial neural network.

\begin{tabular}{|c|c|c|c|}
\hline Fault & Result & Based on SCADA Data & Based on Vibration Data \\
\hline \multirow{3}{*}{ Gear Bearing } & Correct Prediction & $72.5 \%$ & $63 \%$ \\
\cline { 2 - 4 } & Missed Failure & $20 \%$ & $13 \%$ \\
\cline { 2 - 4 } & False Positive & $7.5 \%$ & $25 \%$ \\
\hline \multirow{3}{*}{ Gear Tooth } & Correct Prediction & $75 \%$ & $100 \%$ \\
\cline { 2 - 4 } & Missed Failure & $13 \%$ & $0 \%$ \\
\cline { 2 - 4 } & False Positive & $12 \%$ & $0 \%$ \\
\hline
\end{tabular}

Table 2: Results of two-class neural network predictions for SCADA and vibration data

\subsection{Data groupings}

When training the machine learning algorithm for the gear bearing issue in Section 3.3, SCADA data from 1 month, 2 months, 3 months, 6 months before failure, and healthy (greater than 1 year) were used. However, when testing the algorithm only data from less than one month before failure and greater than 1 year before failure (Healthy) were used. To further test the algorithm, SCADA data from two months before failure, 3 months before failure and 6 months before failure were also used to determine the algorithms ability to correctly predict failures. As seen in Table 3 there are four category columns and four score columns. Each category column heading indicates the data grouping for training the artificial neural network. For example, "1 Month vs Healthy" means the algorithm was trained with only those two data groupings, whereas "1 Month vs 2 Months to Healthy" means the algorithm was trained with 1 month to failure data as the first class and then the combination of the data from 2 months, 3 months, six months and 1 year before failure 
grouped as the second "healthy" class. Unbalanced data means the number of training samples in the "healthy" section out-weigh the number of "1 Month" before failure samples. "Balanced" means the number of training samples in both classes are equal. Each row then provides the percentage of correct predictions, missed failures of false positives when tested with data from 1 month before failure, 2 months before failure, 3 months before failure, 6 months before failure and greater than 1 year before failure (Healthy).

Up to this point in this paper the algorithms have been trained using the groupings highlighted in red in Table 3 "1 Month vs 2 Months to Healthy". It can be seen from Table 3 that when the same groupings are used with unbalanced data (more "healthy" examples than 1 month samples) the Neural Network then has a bias towards "Healthy Predictions". While this leads to a high number of correct predictions when the "Healthy" data is tested, it leads to a very high number for missed failures, $79.2 \%$ when " 1 Month" before failure data is tested. From the 5 groupings tested the "1 Month vs 2 Months to Healthy (Balanced Data)" grouping provides the best performance in terms of maximized correct predictions and minimized missed failures or false positives. Consequently, this grouping has been used throughout this paper.

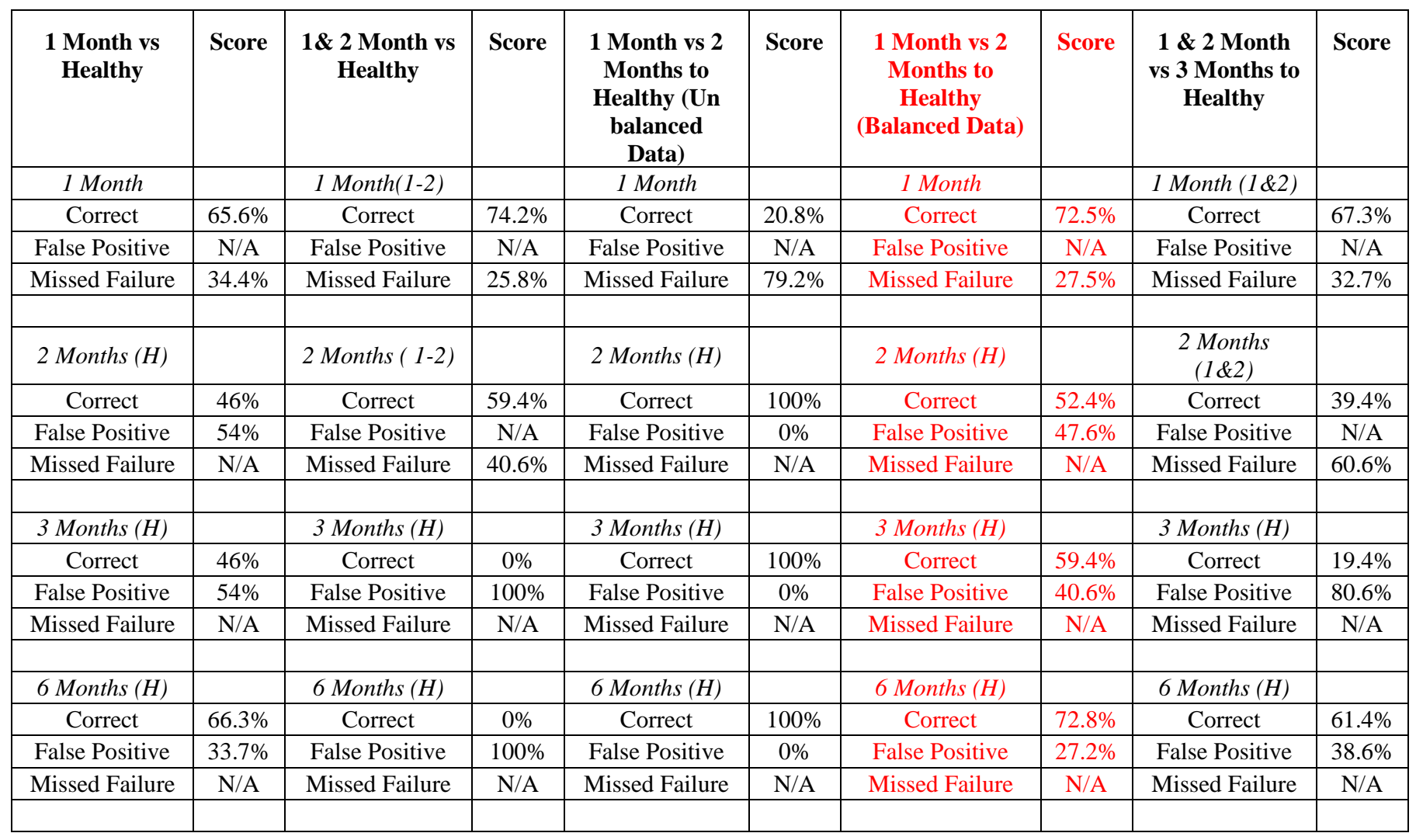




\begin{tabular}{|c|c|c|c|c|c|c|c|c|c|}
\hline Healthy & & Healthy & & Healthy & & Healthy & & Healthy & \\
\hline Correct & $77.5 \%$ & Correct & $14.6 \%$ & Correct & $100 \%$ & Correct & $75.7 \%$ & Correct & $66.4 \%$ \\
\hline Missed Failure & N/A & Missed Failure & N/A & Missed Failure & N/A & Missed Failure & N/A & Missed Failure & N/A \\
\hline
\end{tabular}

Table 3: Results of different data groupings for two class neural network predictions with SCADA data

\subsection{Input weightings}

The results of the weighting analysis described in Section 2.5 are shown below where inputs are ranked in order of importance. As expected, the gearbox temperature sensors, and their delta readings, located closest to the planetary stage bearing where the failure occurred, carry the greatest weight in predicting failure. Gear oil temperature however, is not ranked highly. This indicates that little or no temperature change from the bearing issue is picked up by the gear oil sensors.

1. Delta Bearing 1 Temp and Nac Temp

2. Delta Bearing 1 Temp and Amb Temp

3. Bearing 1 Temp

4. Delta Bearing 2 Temp and Nac Temp

5. Delta Bearing 3 Temp and Nac Temp

6. Delta Bearing 4 Temp and Nac Temp

7. Delta Bearing 2 Temp and Amb Temp

8. Bearing 3 Temp

9. Bearing 2 Temp

10. Delta Bearing 4 Temp and Amb Temp

11. Delta Bearing 3 Temp and Amb Temp

12. Bearing 4 Temp

13. Delta Bearing 5 Temp and Nac Temp

14. Delta Bearing 5 Temp and Amb Temp
15. Average Wind Speed

16. Total Production

17. Bearing 5 Temp

18. Delta Gear Oil Temp 1 and Amb Temp

19. Gear Oil Temp 1

20. Delta Gear Oil Temp 2 and Nac Temp

21. Amb Temp Avg

22. Delta Gear Oil Temp 2 and Amb Temp

23. Delta Gear Oil Temp 1 and Nacelle Temp

24. Nac Temp Avg

25. Gear Oil Temp 2

26. Delta Gear Oil Temp 2 and Gear Oil temp 1

27. Rtr RPM Avg

The accuracy of the model for the different number of inputs is illustrated in Figure 11, the numbers on the $\mathrm{x}$-axis match the numbers in the previous ranking. The features are removed one by one starting with the least important. Figure 11 shows that the accuracy of the overall predictions drop slowly at first while the features of less importance are removed. As the most important 7 or 8 features are removed the graph displays a faster incline in prediction accuracy. 


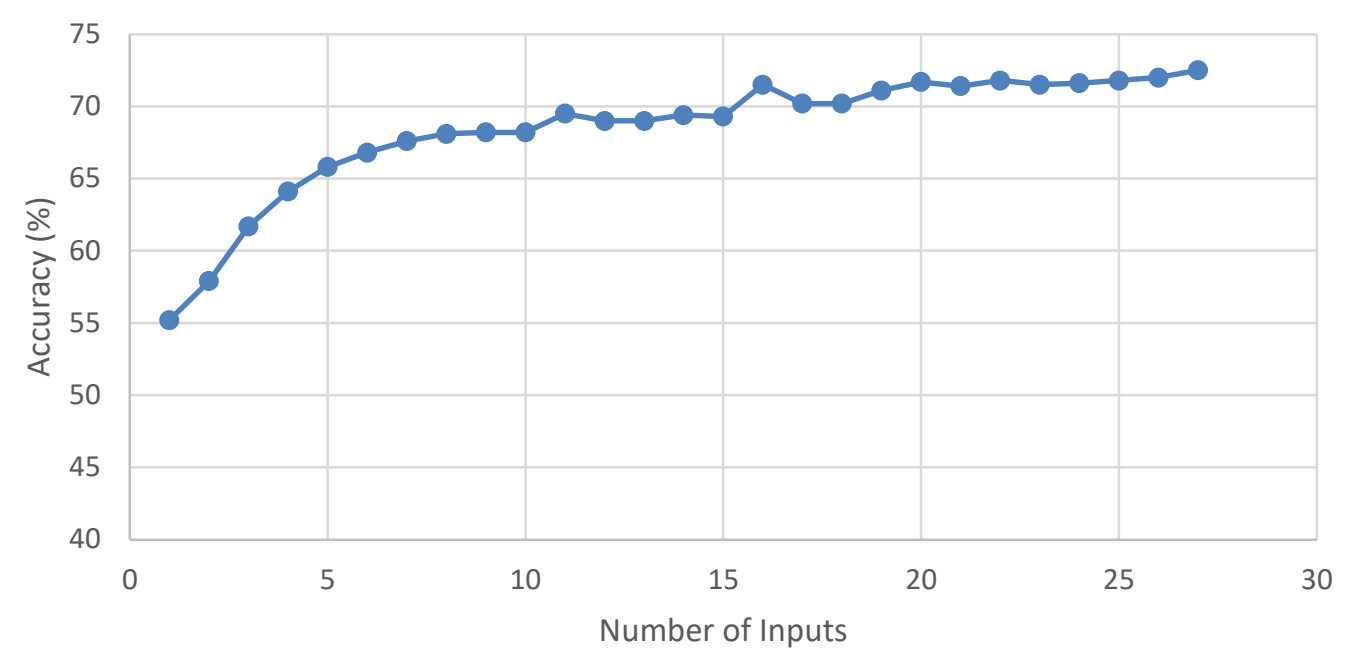

Figure 11: Accuracy of neural network predictions as the number of SCADA data input vary. (Figure created using SCADA data from gear bearing issue)

\begin{tabular}{|c|c|c|c|c|c|c|c|}
\hline $\begin{array}{c}\text { \# of } \\
\text { Inputs }\end{array}$ & $\begin{array}{c}\text { Correct } \\
\text { Predictions }\end{array}$ & $\begin{array}{c}\text { \# of } \\
\text { Inputs }\end{array}$ & $\begin{array}{c}\text { Correct } \\
\text { Predictions }\end{array}$ & $\begin{array}{c}\text { \# of } \\
\text { Inputs }\end{array}$ & $\begin{array}{c}\text { Correct } \\
\text { Predictions }\end{array}$ & $\begin{array}{c}\text { \# of } \\
\text { Inputs }\end{array}$ & $\begin{array}{c}\text { Correct } \\
\text { Predictions }\end{array}$ \\
\hline $\mathbf{2 7}$ & $\mathbf{7 2 . 5 \%}$ & $\mathbf{2 6}$ & $\mathbf{7 2 \%}$ & $\mathbf{2 5}$ & $71.8 \%$ & $\mathbf{2 4}$ & $\mathbf{7 1 . 6 \%}$ \\
\hline $\mathbf{2 3}$ & $71.5 \%$ & $\mathbf{2 2}$ & $\mathbf{7 1 . 8 \%}$ & $\mathbf{2 1}$ & $71.4 \%$ & $\mathbf{2 0}$ & $71.7 \%$ \\
\hline $\mathbf{1 9}$ & $71.1 \%$ & $\mathbf{1 8}$ & $70.2 \%$ & $\mathbf{1 7}$ & $70.2 \%$ & $\mathbf{1 6}$ & $71.5 \%$ \\
\hline $\mathbf{1 5}$ & $69.3 \%$ & $\mathbf{1 4}$ & $69.4 \%$ & $\mathbf{1 3}$ & $69 \%$ & $\mathbf{1 2}$ & $69 \%$ \\
\hline $\mathbf{1 1}$ & $69.5 \%$ & $\mathbf{1 0}$ & $68.2 \%$ & $\mathbf{9}$ & $68.2 \%$ & $\mathbf{8}$ & $68.1 \%$ \\
\hline $\mathbf{7}$ & $67.6 \%$ & $\mathbf{6}$ & $66.8 \%$ & $\mathbf{5}$ & $65.8 \%$ & $\mathbf{4}$ & $64.1 \%$ \\
\hline $\mathbf{3}$ & $61.7 \%$ & $\mathbf{2}$ & $57.9 \%$ & $\mathbf{1}$ & $55.2 \%$ & $\mathbf{0}$ & $0 \%$ \\
\hline
\end{tabular}

Table 4: Results of different number of inputs for two class neural network predictions with SCADA data for bearing issue

\subsection{Multi-class artificial neural network prediction}

Table 5 shows the results from the training of a multi-class neural network for both the gear bearing and gear tooth issue using both SCADA and vibration data to predict if those failure modes will occur within month 0-1, 1-2, 2-3 or outside 3 months (labeled as healthy) for SCADA data. For vibration data it was labeled as month 1-2, 5-6 or greater than 1 year (labeled as healthy). The 3-4 month and 6-12 month intervals were not included in the vibration based models due to a data acquisition and storage issue. The high frequency vibration data was greater than $25 \mathrm{kHz}$ so with such large amounts of data to be acquired, stored and analyzed the authors had to be selective in the months they used. For data reduction purposes the authors sampled the data from a time period close to failure (1-2 months) a healthy point (Over 1 year to failure) and a point in 
between (5-6 months). Correct predictions shown in Table 5 are highlighted across the diagonal of each confusion matrix. It can be seen that the multi-class neural network is most successful in predicting a gear tooth failure using vibration data. The number of correct predictions starts to drop for the gear bearing issue when vibration data is used because of the failure feature extraction issue in the high frequency data as detailed in Section 3.2. For both failure modes the multi-class neural networks trained with SCADA data struggle to predict failures correctly, particularly in months 2 and 3 . One reason for this may be that the 10minute averaged SCADA readings have little or no differences in months previous to one month before failure. For example, the failure issue may not show itself in the 10-minute averaged data so clearly until roughly 1 month before failure.

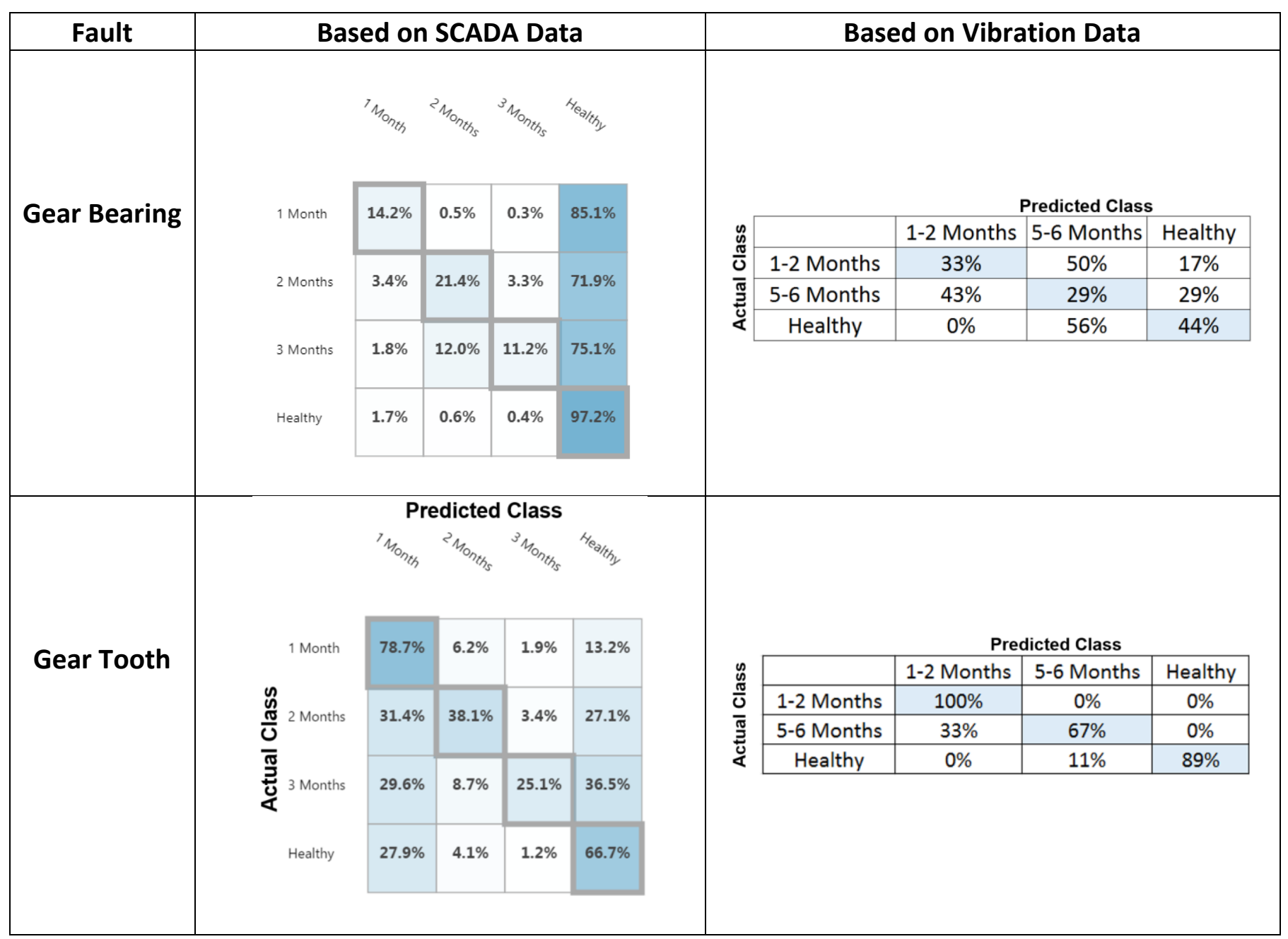

Table 5: Results of multi-class class neural network predictions for SCADA and Vibration data

\section{Conclusion}


This section will conclude each of the sub-sections outlined in Results and Discussion, Section 3.

This work found that trends in 10-minute SCADA oil or bearing temperature data showed no indication of failure when considered alone. Through analysis of thousands of wind turbines with hundreds of gearbox tooth or bearing failures it was shown that temperatures could be lower in the month before failure than over a year before failure depending on a turbine's power production in that period. Consequently, it was concluded that rather than simple temperature trending in isolation, algorithms that consider a larger number of SCADA data inputs such as power production, generator RPM, rotor RPM, bearing temperature and so on were required to correctly predict failure and remaining useful life.

Through analysis of gearbox vibration data in the build up to gearbox failure related to gear tooth issues this work showed that trends (side bands around gear meshing frequencies) could be clearly seen in the data, six to seven months before failure occurred. While trends could be seen for gear tooth issues, to date, this work did not discover trends in the vibration data in the build up to gearbox planetary stage bearing issues.

In this paper, three machine learning algorithms were trained and tested to determine which provided the most correct predictions and lowest missed failures/false positives when trained with SCADA data in the build up to gearbox bearing failures. This paper found that an artificial neural network out performed both support vector machines (with a linear kernel) and logistic regression for the above purpose.

A two class neural network was trained and tested using both SCADA data and vibration data for failure prediction of a planetary gear bearing and an intermediate stage gear tooth. Results showed that a neural network is the most successful in predicting an intermediate gear tooth failure using vibration data, this is followed by predicting the same gear tooth issue using SCADA data. It can be concluded that the use of vibration data provides the most correct predictions when clear features can be extracted from the spectrum (as in the gear tooth issue in this paper). In this paper a lower number of correct failure predictions were seen for the gear bearing issue based on vibration data because of the lack of clear feature extraction from the spectrum. 
When training two class neural networks based on wind turbine data, a number of different groupings can be used. For a two class prediction this paper found that the most successful method for grouping the training data is to group SCADA data that is recorded one month before failure as the first class and the second class is represented by SCADA data that is recorded at points in 2 months, 3 months, 6 months and greater than 1 year before failure. Both the first class and the second class mentioned above should be balanced in terms of the amount of data used to train the algorithm to ensure the most correct predictions.

The SCADA data input weighting analysis in this paper showed that the gearbox bearing temperatures, and their deltas that are closest to the failing planetary stage bearing carry the most weight in predicting the failure out of all of the inputs examined. The gear oil temperatures are less important suggesting this failure mode does not influence the gearbox oil in manner that the algorithm identifies as indicative of failure.

Lastly this paper shows results from the training of a multi-class artificial neural network for both the gear bearing and gear tooth issue using both SCADA and vibration data. This work found that multi-class classification was possible for predicting failure and remaining useful life but was more successful with vibration data than SCADA data.

\section{Acknowledgements}

This work was funded by the Engineering and Physical Sciences Research Council EP/G037728/1

\section{References}

[1] Bakhshi R, Sandborn P. A Return on Investment Model for the Implementation of New Technologies on Wind Turbines IEEE Transactions on Sustainable Energy 2017

[2] Department of Energy and Climate Change UK. Electricity Generation Costs 2013

[3] Crabtree C, Zappala D, Hogg S. Wind energy: UK experiences and offshore operational challenges Power and Energy 2015, Vol. 229(7) 727746 IMechE 2015

[4] Bach-Andersen M, Rømer-Odgaard B, Winther O. Deep learning for automated drivetrain fault detection. Wind Energy, 21(1):29-41, January 2018. 
[5] Lau BCP, Ma EWM, Pecht M. Review of offshore wind turbine failures and fault prognostic methods. In Proceedings of the IEEE Prognostics and System Health Management Conference, pages 1-5, May 2012.

[6] Feng Y, Qiu Y, Crabtree CJ, Long H, Tavner PJ. Monitoring wind turbine gearboxes. Wind Energy, 16(5):728-740, 2013.

[7] Qiao W, Lu D. A survey on wind turbine condition monitoring and fault diagnosis — Part I: Components and subsystems. IEEE Transactions on Industrial Electronics, 62(10):6536-6545, Oct 2015.

[8] Qiao W, Lu D. A survey on wind turbine condition monitoring and fault diagnosis — Part II: Signals and signal processing methods. IEEE Transactions on Industrial Electronics, 62(10):6546-6557, October 2015.

[9] Qiao W, Zhang P, Chow MY. Condition monitoring, diagnosis, prognosis, and health management for wind energy conversion systems. IEEE Transactions on Industrial Electronics, 62(10):6533-6535, October 2015.

[10] Zaher A, McArthur S, Infield D, Patel Y. Online wind turbine fault detection through automated SCADA data analysis. Wind Energy, 12(6):574-593, September 2009.

[11] Carroll J, McDonald A, Dinwoodie I, McMillan D, Revie M, Lazakis I. Availability, operation and maintenance costs of offshore wind turbines with different drive train configurations. Wind Energy, 20(2):361-378, February 2017.

[12] Bangalore P, Letzgus S, Karlsson D, Patriksson M. An artificial neural network based condition monitoring method for wind turbines, with application to the monitoring of the gearbox. Wind Energy, 20(8):1421-1438, August 2017.

[13] Cao M, Qiu Y, Feng Y, Wang H, Li D. Study of wind turbine fault diagnosis based on unscented Kalman filter and SCADA data. Energies, 9(10):1-18, 2016.

[14] Cheng F, Qu L, Qiao W. Fault prognosis and remaining useful life prediction of wind turbine gearboxes using current signal analysis. IEEE Transactions on Sustainable Energy, 9(1):157-167, January 2018.

[15] Qian P, Ma X, Cross P. Integrated data-driven model-based approach to condition monitoring of the wind turbine gearbox. IET Renewable Power Generation, 11(9):1177-1185, September 2017.

[16] Qiu Y, Chen L, Feng Y, Xu Y. An approach of quantifying gear fatigue life for wind turbine gearboxes using supervisory control and data acquisition data. Energies, 10(8), 2017.

[17] Qiu Y, Feng Y, Sun J, Zhang W, Infield D. Applying thermophysics for wind turbine drivetrain fault diagnosis using scada data. IET Renewable Power Generation, 10(5):661-668, 2016.

[18] Sadeghi MH, Raflee J, Arvani F, Harifi A. A fault detection and identification system for gearboxes using neural networks. In 2005 International Conference on Neural Networks and Brain, volume 2, pages 964-969, October 2005.

[19] Strączkiewicz M, Barszcz T. Application of artificial neural network for damage detection in planetary gearbox of wind turbine. Shock and Vibration, 2016:1-12, 2016. 
[20] Wang L, Zhang Z, Long H, Xu J, Liu R. Wind turbine gearbox failure identification with deep neural networks. IEEE Transactions on Industrial Informatics, 13(3):1360-1368, June 2017.

[21] Zappala D, Tavner PJ, Crabtree CJ, Sheng S. Side-band algorithm for automatic wind turbine gearbox fault detection and diagnosis. IET Renewable Power Generation, 8(4):380-389, May 2014.

[22] Zhao W, Siegel D, Lee J, Su L. An integrated framework of drivetrain degradation assessment and fault localization for offshore wind turbines. International Journal of Prognostics and Health Management, 4:46$58,2013$.

[23] Rafiee J, Arvani F, Harifi A, Sadeghi MH. Intelligent condition monitoring of a gearbox using artificial neural network. Mechanical Systems and Signal Processing 2006

[24] Tian Z. An artificial neural network method for remaining usful life prediction of equipment subject to condition monitoring J Intell. Manuf. 2012

[25] Garcia M, Sanz Bobi M, del Pico J. SIMAP: Intelligent System for Predictive Maintenance Application to the Health Condition Monitoring of a Wind Turbine Gearbox Computers in Industry 2016

[26] Gao R, Wang J, Yan R. Defect Diagnosis in Wind Turbine Gearbox based on Sideband Energy and Enveloping Spectral Analysis. NREL Round Robin 2012

[27] Tamilselvan P, Wang P, Sheng S, Twomey J. A Two-Stage Diagnosis Framework for Wind Turbine Gearbox Condition Monitoring. NREL Round Robin 2012

[28] Microsoft Azure. A-Z List of Machine Learning Studio Modules. Accessed at: https://msdn.microsoft.com/en-us/library/azure/dn906033.aspx. Accessed on 10/08/2017

[29] Jena M, Samantaray S. Data-mining-based intelligent differential relaying for transmission lines including UPFC and wind farms. IEEE Transactions on Neural Networks and Learning Systems 2016

[30] Microsoft Azure: Permutation Feature Importance. Accessed at: https://msdn.microsoft.com/enus/library/azure/dn997262.aspx Accessed on 06/08/2017

[31] Satizábal H, Pérez-Uribe A. Relevance Metrics to Reduce Input Dimensions in Artificial Neural Networks. ICANN 2007

[32] Feng Y, Qiu Y, Crabtree C, Long H, Tavner P. Use of SCADA and CMS Signals for Failure Detection and Diagnosis of Wind Turbine Gearbox. EWEA 2011

[33] Cheng F, Wang J, Qu L, Qiao W. Rotor-Current-Based Fault Diagnosis for DFIG Wind Turbine Drivetrain Gearboxes Using Frequency Analysis and a Deep Classifier. IEEE Trans. On Industry Applications Vol 54 No.2 April 2018 